\title{
The novel norovirus genotype Gll.17 is the predominant strain in diarrheal patients in Shanghai, China
}

\author{
Lifeng Pan ${ }^{1,2,3 \dagger}$, Caoyi Xue ${ }^{1,2,4 \dagger}$, Huiqin Fu ${ }^{2,3}$, Dan Liu ${ }^{2,3}$, Linying Zhu ${ }^{1,2,3}$, Chang Cui ${ }^{1,2,4}$, Weiping Zhu ${ }^{1,2,4^{*}}$, \\ Yifei $\mathrm{Fu}^{1,2}$ and Sun Qiao ${ }^{1,2^{*}}$
}

\begin{abstract}
In the winter of 2014-2015, a novel norovirus (NoV) strain (GII.17) was reported to be the major cause of gastroenteritis outbreaks in East Asia. To determine the time course of gastroenteritis infections associated with the Gll.17 strain and whether Gll.17 was the main epidemic strain in diarrheal patients in Shanghai, 2169 stool samples were collected and tested. The detection rate of NoV GI and GII NoV strains was 0.83 and $24.02 \%$, respectively. Phylogenetic analysis confirmed that there were seven NoV genotypes, among which GII.4 and GII.17 were the main genotypes. The Gll.17 strain was first detected in a sample collected on August 14th, 2014, and beginning in January 2015, the novel Gll.17 strain replaced the GIl.4 strain as the dominant NoV genotype causing acute gastroenteritis in patients in Shanghai.
\end{abstract}

Norovirus $(\mathrm{NoV})$ is a leading cause of diarrhea inpatients of all age groups with acute gastroenteritis worldwide [1]. Starting in November 2014, Guangdong [2], Jiangsu [3], and Zhejiang [4] in China, and Japan [5] reported diarrheal disease outbreaks that were caused by a novel NoV genotype GII.17. Chen et al. [6] reported this novel genotype collected from sporadic patients in Shanghai during the winter of 2014-2015 has evolutionary relationships with other reported genotypes $[2,5]$. Here, we characterized the molecular epidemiology of NoV infections in Shanghai between July 2014 and June 2015 to examine when did this novel NoV genotype strain begin to prevalent and whether the NoV GII.4 is still the leading NoV genotype in Shanghai, China.

A total of 2169 stool samples were collected from 18,669 diarrheal patients, reported to the syndromic surveillance network from 12 sentinel hospitals, between July 2014 and June 2015 . The detection rate of the NoV

\footnotetext{
*Correspondence: wpzhu@pdcdc.sh.cn; q_sun@pdcdc.sh.cn ${ }^{\dagger}$ Lifeng Pan and Caoyi Xue contributed equally to this work

${ }^{1}$ Research Base of Key Laboratory of Surveillance and Early Warning on Infectious Disease in China CDC, Shanghai Pudong New Area Center for Disease Control and Prevention, 3039 Zhangyang Road, Shanghai 200136, China

Full list of author information is available at the end of the article
}

GI and GII was $0.83 \%(18 / 2169)$ and $24.02 \%(521 / 2169)$ by real-time reverse transcription polymerase chain reaction (qRT-PCR), respectively.

Partial nucleotide sequences of the NoV GII ORF2 gene was amplified. Genotypes were determined using Norovirus Genotyping Tool Version 1.0. The numbers of strains corresponding to the GII.2, GII.3, GII.4, GII.6, GII.13, GII.17 and GII.21 strains were 3, 5, 133, 3, 8, 286 and 10, respectively. GII.17 (63.84\%, 286/448) and GII.4 $(29.69 \%, 133 / 448)$ were the most prevalent NoV GII genotypes between July 2014 and January 2015.

NoV infections occurred in all seasons and peaked between October 2014 and April 2015 (Fig. 1). The novel genotype GII.17 strain was first detected in a 24-year-old female patient on August 14th, 2014 in Shanghai. Beginning in January 2015, GII.17, which clustered to the novel variant Kawasaki_2014, was the most prevalent genotype of NoV GII, replacing GII.4 as the most prevalent NoV genotype in Shanghai. After May 2015, the GII.4 strain was rarely detected (Fig. 1).

As the most prevalent strain, the GII.4 genotype Sydney_2012 and its variants were surveyed and reported globally [7-12], beginning in 2012 [13]. However, starting in November 2014, a novel NoV genotype GII.17 was reported to cause diarrheal disease outbreaks, 


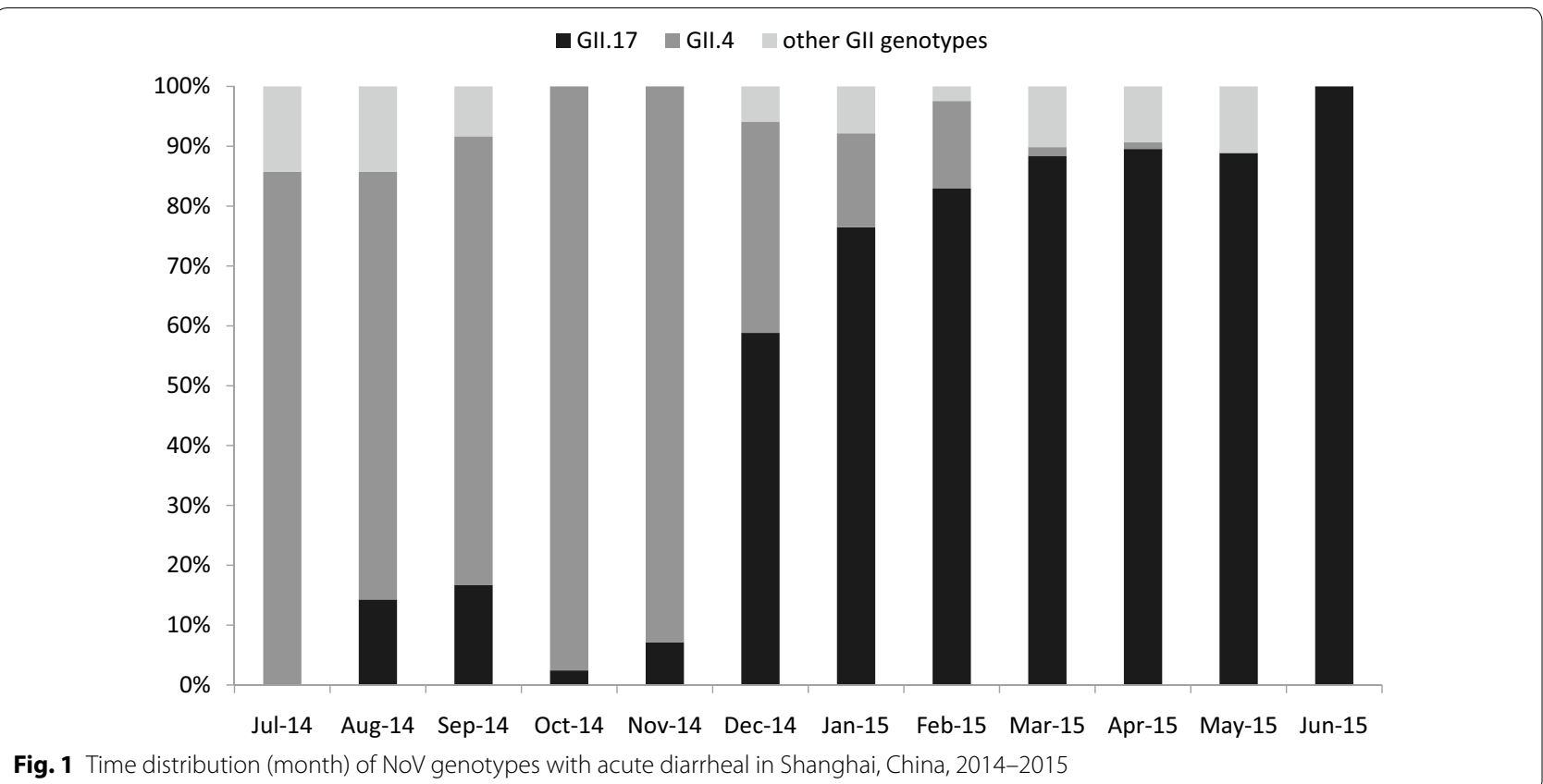

Fig. 1 Time distribution (month) of NoV genotypes with acute diarrheal in Shanghai, China, 2014-2015

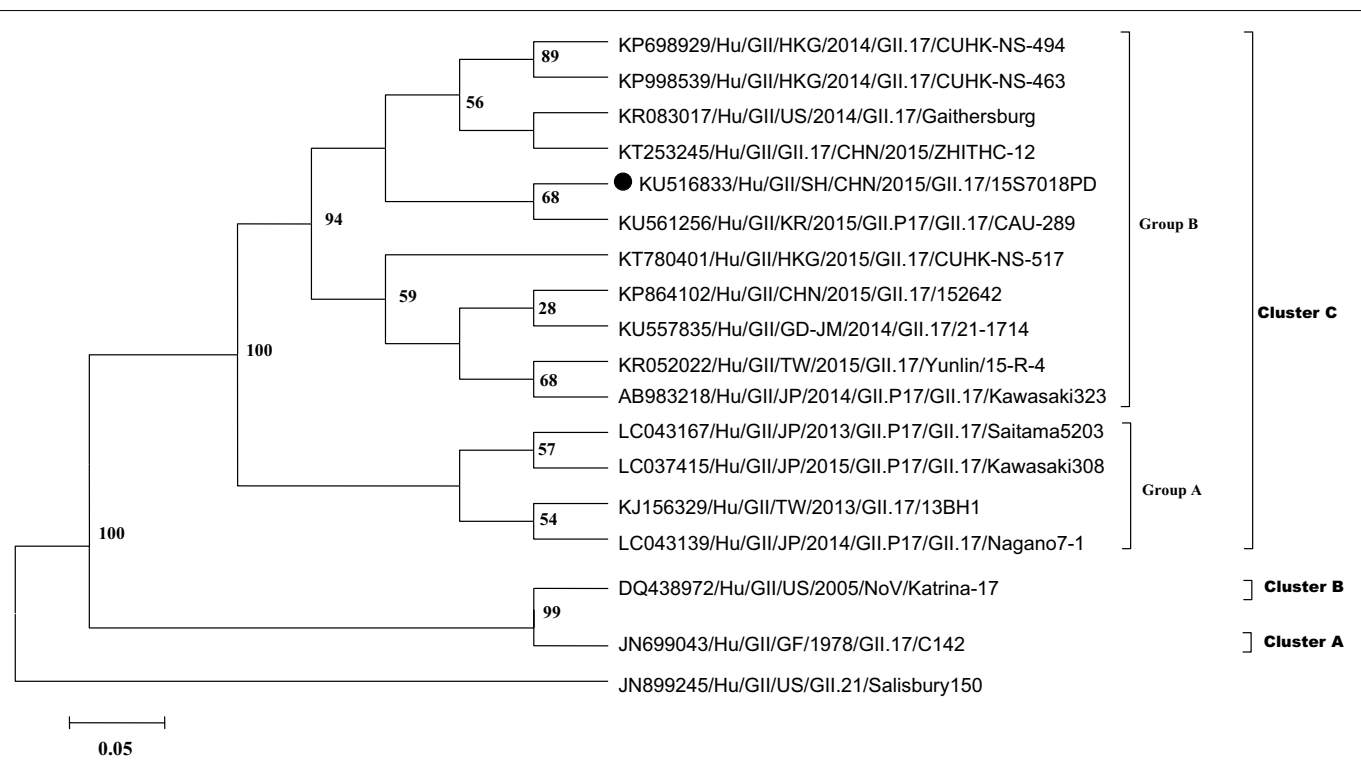

Fig. 2 Phylogenetic analysis of partial capsid region (ORF2) of NoV strains. Presented is a cladogram with supporting bootstrap values with full length VP1 nucleotide of reference NoVs according to LeBlanc et al. [15]. Representative strain from acute diarrheal patients in Shanghai, 2014-2015 are shown with black circle

worldwidely [2, 5, 14-16] and Matesushiam et al. [5] found that this novel genotype had a polymerase sequence and amino acid substitutions in the capsid region. In this study, we reported that the GII.17 genotype was first detected in diarrheal patients in August 2014, which was earlier than the first reported outbreak in Jiangsu [3]. Similar with the findings by Chen et al.
[6], the GII.17 genotype became the predominant genotype beginning in November 2014 (Fig. 2). Our findings demonstrate that beginning in December 2014, GII.17 replaced GII.4 as the most prevalent NoV genotype in Shanghai. Teasing out viral, environmental, or host specific factors should be investigated to understand emergence of GII.17 [15] and ongoing surveillance should be 
performed to verify whether this novel genotype would be prevalent continuously.

\section{Abbreviations}

NoV: norovirus; SPV: sapovirus; RTV: rotavirus; ADV: adenovirus; ASTV: astrovirus; NGTV 1: Norovirus Genotyping Tool Version 1.0; qRT-PCR: real-time reverse transcription polymerase chain reaction; RT-PCR: reverse transcription polymerase chain reaction; ORF2: open reading frame 2 .

\section{Authors' contributions}

$L P$ and $Y F$ conceived and designed the experiments. HF, DL and $L Z$ performed the experiments. CX and CC performed the statistical analysis. WZ, YF and QS conceived the study. LP and XY wrote the manuscript. All authors read and approved the final manuscript.

\section{Author details \\ ${ }^{1}$ Research Base of Key Laboratory of Surveillance and Early Warning on Infec- tious Disease in China CDC, Shanghai Pudong New Area Center for Disease Control and Prevention, 3039 Zhangyang Road, Shanghai 200136, China. \\ 2 Pudong Institute of Preventive Medicine, Fudan University, 3039 Zhangyang Road, Shanghai 200136, China. ${ }^{3}$ Microbe Test Section, Pudong New Area Center for Disease Control and Prevention, 3039 Zhangyang Road, Shang- hai 200136, China. ${ }^{4}$ Department of Infectious Disease, Pudong New Area Center for Disease Control and Prevention, 3039 Zhangyang Road, Shang- hai 200136, China.}

\section{Acknowledgements}

We thank the staff of 12 sentinel hospitals in the present study.

\section{Competing interests}

The authors declare that they have no competing interests.

\section{Availability of data and materials}

The database supporting the conclusions of this article is included within the article's Additional file.

\section{Ethics approval and consent to participate}

The study protocol was in accordance with the ethical guidelines of the 1975 Declaration of Helsinki and was approved by the Review Board at Shanghai Pudong New Area Center for Disease Control and Prevention.

\section{Funding}

This work was supported in part by Key Discipline Construction in Pudong New Area Health System (No.PWzx2014-14), Pudong New Area Science and Technology Development-Innovation Fund (No. PKJ2013-Y39) and Research Grant for Health Science and Technology of Pudong Health and Family Planning of Shanghai (Grant No. PW2015B-4). The funder had no role in the study design, data collection and analysis, decision to publish or preparation of the manuscript.

Received: 4 July 2016 Accepted: 12 October 2016 Published online: 26 October 2016

\section{References}

1. Patel MM, Widdowson MA, Glass RI, Akazawa K, Vinje J, Parashar UD. Systematic literature review of role of noroviruses in sporadic gastroenteritis. Emerg Infect Dis. 2008;14(8):1224-31.
2. Lu J, Sun L, Fang L, Yang F, Mo Y, Lao J, Zheng H, Tan X, Lin H, Rutherford S, et al. Gastroenteritis outbreaks caused by norovirus Gll.17, Guangdong Province, China, 2014-2015. Emerg Infect Dis. 2015;21(7):1240-2.

3. Fu J, Ai J, Jin M, Jiang C, Zhang J, Shi C, Lin Q, Yuan Z, Qi X, Bao C, et al. Emergence of a new Gll.17 norovirus variant in patients with acute gastroenteritis in Jiangsu, China, September 2014 to March 2015. Euro Surveill. 2015;20(24):21157.

4. Han J, Ji L, Shen Y, Wu X, Xu D, Chen L. Emergence and predominance of norovirus Gll.17 in Huzhou, China, 2014-2015. Virol J. 2015;12:139.

5. Matsushima Y, Ishikawa M, Shimizu T, Komane A, Kasuo S, Shinohara M, Nagasawa K, Kimura H, Ryo A, Okabe N, et al. Genetic analyses of Gll.17 norovirus strains in diarrheal disease outbreaks from December 2014 to March 2015 in Japan reveal a novel polymerase sequence and amino acid substitutions in the capsid region. Euro Surveill. 2015;20(26):21173.

6. Chen H, Qian F, Xu J, Chan M, Shen Z, Zai S, Shan M, Cai J, Zhang W, He J, et al. A novel norovirus Gll.17 lineage contributed to adult gastroenteritis in Shanghai, China, during the winter of 2014-2015. Emerg Microb Infect. 2015;4(11):e67.

7. Wu FT, Chen HC, Yen C, Wu CY, Katayama K, Park Y, Hall AJ, Vinje J, Huang JC, Wu HS. Epidemiology and molecular characteristics of norovirus Gll.4 Sydney outbreaks in Taiwan, January 2012-December 2013. J Med Virol. 2015;87(9):1462-70.

8. Manso CF, Romalde JL. Molecular epidemiology of norovirus from patients with acute gastroenteritis in northwestern Spain. Epidemiol Infect. 2015;143(2):316-24.

9. Huo Y, Cai A, Yang H, Zhou M, Yan J, Liu D, Shen S. Complete nucleotide sequence of a norovirus Gll.4 genotype: evidence for the spread of the newly emerged pandemic Sydney 2012 strain to China. Virus Genes. 2014;48(2):356-60.

10. Polkowska A, Ronnqvist M, Lepisto O, Roivainen M, Maunula L, Huusko S, Toikkanen S, Rimhanen-Finne R. Outbreak of gastroenteritis caused by norovirus GIl.4 Sydney variant after a wedding reception at a resort/activity centre, Finland, August 2012. Epidemiol Infect. 2014;142(9):1877-83.

11. Hoa Tran TN, Trainor E, Nakagomi T, Cunliffe NA, Nakagomi O. Molecular epidemiology of noroviruses associated with acute sporadic gastroenteritis in children: global distribution of genogroups, genotypes and GIl.4 variants. J Clin Virol. 2013;56(3):185-93.

12. Inaida S, Shobugawa $Y$, Matsuno S, Saito R, Suzuki H. The South to north variation of norovirus epidemics from 2006-07 to 2008-09 in Japan. PLoS ONE. 2013;8(8):e71696

13. Eden JS, Tanaka MM, Boni MF, Rawlinson WD, White PA. Recombination within the pandemic norovirus Gll.4 lineage. J Virol. 2013;87(11):6270-82.

14. Khamrin P, Kumthip K, Yodmeeklin A, Supadej K, Ukarapol N, Thongprachum A, Okitsu S, Hayakawa S, Ushijima H, Maneekarn N. Molecular characterization of norovirus Gll.17 detected in healthy adult, intussusception patient, and acute gastroenteritis children in Thailand. Infect Genetics Evol. 2016;44:330-3.

15. LeBlanc JJ, Pettipas J, Gaston D, Taylor R, Hatchette TF, Booth TF, Mandes R, McDermid A, Grudeski E. Outbreak of norovirus GII.P17-GIl.17 in the Canadian Province of Nova Scotia. Can J Infect Dis Med Microbiol. 2016;2016:1280247.

16. Parra Gl, Green KY. Genome of emerging norovirus Gll.17, United States, 2014. Emerg Infect Dis. 2015;21(8):1477-9. 